Supporting Information

\title{
Light absorption by organic aerosol emissions rivals that of black carbon from residential biomass fuels in South Asia
}

\author{
Apoorva Pandey ${ }^{1}$, Alice Hsu ${ }^{1}$, Suresh Tiwari ${ }^{2}$, Shamsh Pervez ${ }^{3}$, Rajan K. Chakrabarty ${ }^{1, *}$ \\ ${ }^{1}$ Department of Energy, Environmental and Chemical Engineering, Washington University in St. \\ Louis, Missouri - 63130, USA. \\ ${ }^{2}$ Indian Institute of Tropical Meteorology, Pune 411008, India \\ ${ }^{3}$ School of Studies in Chemistry, Pandit Ravishankar Shukla University, Raipur, Chhattisgarh \\ 492010, India \\ * Correspondence to: chakrabarty@wustl.edu
}




\section{Methods:}

1. Field sampling: Experimental methods for the field study conducted between December $19^{\text {th }}$ and $30^{\text {th }}$ of 2015 in Chhattisgarh, India have previously been discussed in Pandey, et al. ${ }^{1}$. Test fuels included fuelwood, agricultural residue and cattle dung collected from different regions in India. All fuels were analyzed for elemental (carbon, oxygen, hydrogen, nitrogen) composition and moisture content. Each cooking test, involving the preparation of a local meal item, was conducted by a member (any one of five) of the research team which included several locally-based researchers familiar with the stove operation. Ignition was initiated using a small amount of dung (20-50 g) doused with $\sim 10 \mathrm{ml}$ kerosene and the test fuel was added after flaming conditions were established. The initial ten-minute period of the combustion cycle was termed the ignition phase. The remainder of the cooking cycle was designated as the flaming phase when a visible flame was present: re-kindling sometimes led to a diminished or nearly extinguished flame and these instances were categorized under ignition. When only glowing embers were present, combustion entered the smoldering phase. Two of the fuels (U.P. dung and Chh. rice straw) could not sustain the flaming phase for more than a few minutes. These fuels were therefore burned in combination with U.P. wood.

The kitchen was a single room on the second floor of the house, separate from all other rooms. A permanently open door and a partially covered window covered were ventilation sources. Real-time instruments sampled naturally diluted emissions from an eight-armed stainless steel placed $\sim 1.2 \mathrm{~m}$ above the top of the stove (Fig. S1). A Testo-350 gas analyser continuously measured carbon monoxide $(\mathrm{CO})$ and carbon dioxide $\left(\mathrm{CO}_{2}\right)$ during the cooking tests. $\mathrm{PM}_{2.5}$ samples were collected on $47 \mathrm{~mm}$ PTFE membrane and quartz fiber filters using Minivol (5 L $\min ^{-1}$ ) samplers (AirMetrics Model 4.2), during different times in each cooking cycle. Filter 
sample durations ranged from 0.5 to 4 minutes to prevent filter overloading. Field blanks were collected (minimum sampling duration of 15 minutes) each day before testing. Wireless particle light scattering sensors (Sharp GP2Y) were attached to the Minivol sampler and the sampling probe during six experiments to check for any significant differences in the particle concentrations measured at the two locations. Measurements where either sensor was saturated were discarded, and a linear regression analysis performed on the valid data points. A correction factor of 1.6 was applied based on regression slope ${ }^{11}$.

2. Filter optical analysis: PTFE filters were weighed before and after sampling using a microbalance at Pt. Ravishankar Shukla University, Raipur, India to obtain the net mass deposited. While in the test kitchen, the filter samples were kept in a portable insulated box with freezer packs. Both sets of filters were stored in a freezer $\left(-20^{\circ} \mathrm{C}\right)$ after each day of sampling. Storage period (before analysis) was less than 50 days for all filters. At the end of the study, the quartz filters were transported to Desert Research Institute, Nevada, where they were analyzed using the Interagency Monitoring of Protected Visual Environments - A (IMPROVE_A) thermal-optical reflectance (TOR) method to determine elemental and organic carbon fractions in the sampled particulates. The PTFE filters were brought to Washington University in St Louis for optical analysis. For each filter, sample-side transmittance $\left(T_{s}\right)$ and reflectance $\left(R_{s}\right)$ were measured for wavelengths $350-900 \mathrm{~nm}$ using a Perkin-Elmer LAMBDA 35 UV-vis spectrophotometer (see Pandey, et al. ${ }^{2}$ for more details on filter analysis technique).

Statistical and error analysis methods: We derived the bounds on all reported quantities from the distribution of values derived from individual samples. All results are reported as means \pm standard deviations or along with 95\% confidence intervals. Given that emissions data are non-negative and exhibit distributions with long tails (due to the occurrence of high 
emissions events ${ }^{1,3}$ ), a lognormal distribution was selected as a probable candidate for representing pooled absorption emission factor (AEF) data. We evaluated the assumption of lognormality of AEF data by performing the Anderson-Darling test on $\log (\mathrm{AEF})$ : test statistic was calculated as 0.324 and the critical value at a significance level of 0.05 was 0.7408 . The null hypothesis (that data are normally distributed) is rejected if the test statistic is larger than the critical value. Thus, in this case the null hypothesis was valid at a 0.05 significance level. Student's $t$-tests comparing AEFs for the three fuel categories (fuelwood, agricultural residue and dung) were also performed on $\log (\mathrm{AEF})$ instead of directly using AEF values.

Figures (S1-S6) and Table (S1):

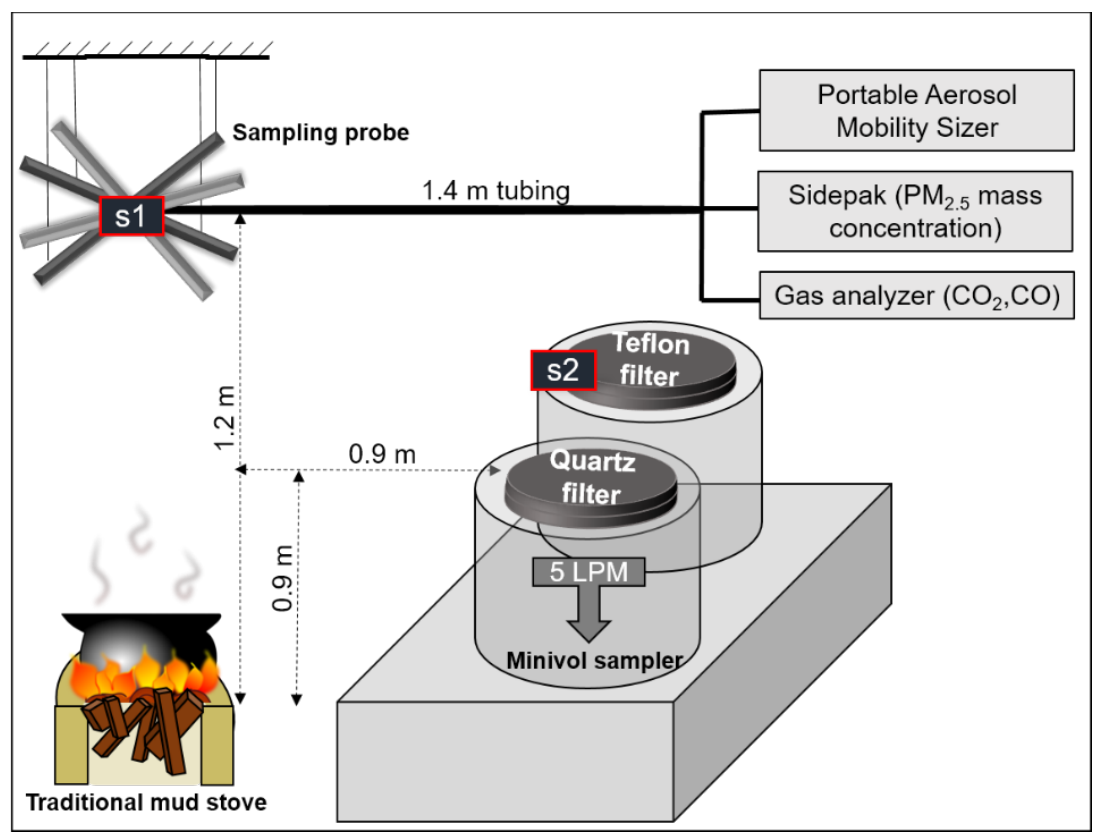

Fig. S1: Schematic representation of the experimental setup. S1 and S2 denote the position of the wireless optical sensors. 


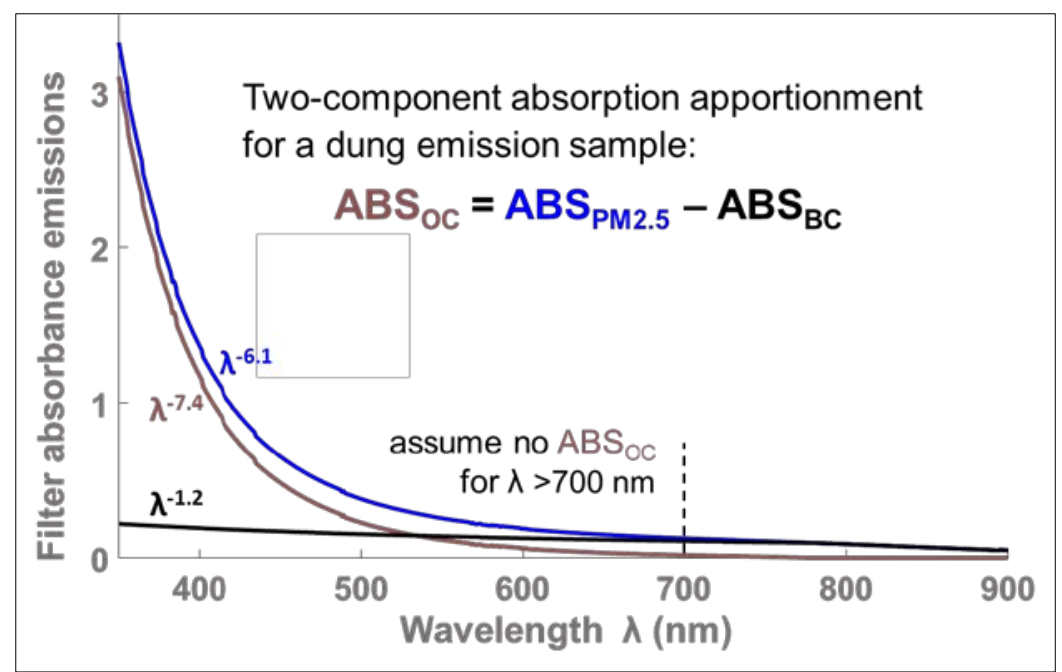

Fig. S2: Absorption spectrum for a sample of dung emissions is deconvoluted by assigning all absorption at wavelengths greater than $700 \mathrm{~nm}$ to $\mathrm{BC}$ and extrapolating $\mathrm{BC}$ absorption at smaller wavelengths using a fixed BC AAE (1.2 in the figure).

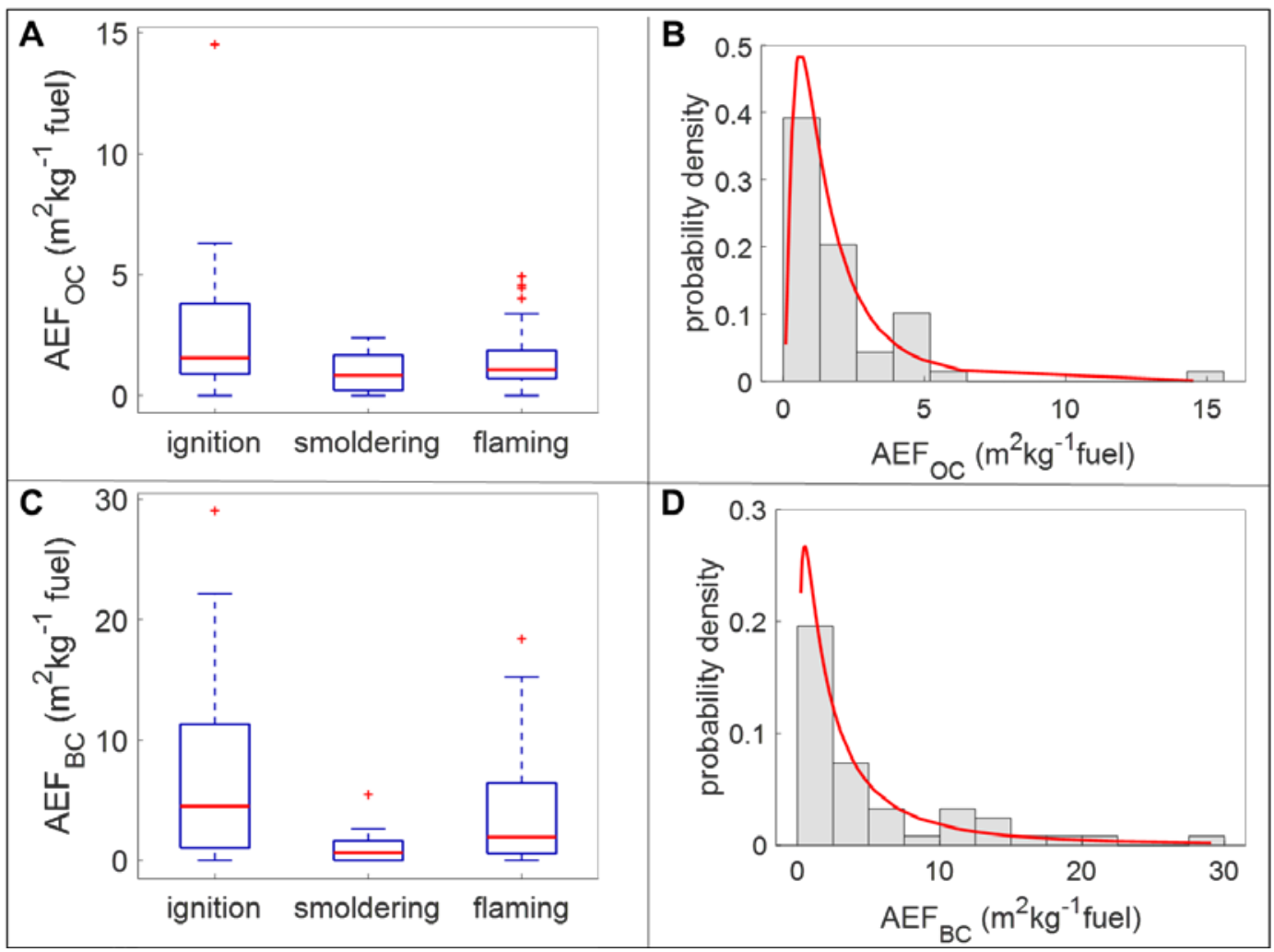


Fig. S3: Absorption emission factors in $\mathrm{m}^{2} \mathrm{~kg}^{-1}$ fuel consumed, at $550 \mathrm{~nm}$ wavelength: (A) AEFoc grouped by observed combustion phase: boxes denote the upper and lower quartiles and whiskers denote 1.5 times the interquartile range, outliers are shown as red + symbols, number of samples for each category are specified above the whiskers and (B) $\mathrm{AEF}_{\mathrm{OC}}$ shown as a histogram of all samples, overlaid by a fitted lognormal distribution. Plots in (C) and (D) are the same as (A) and (B), respectively, but for $\mathrm{AEF}_{\mathrm{BC}}$.
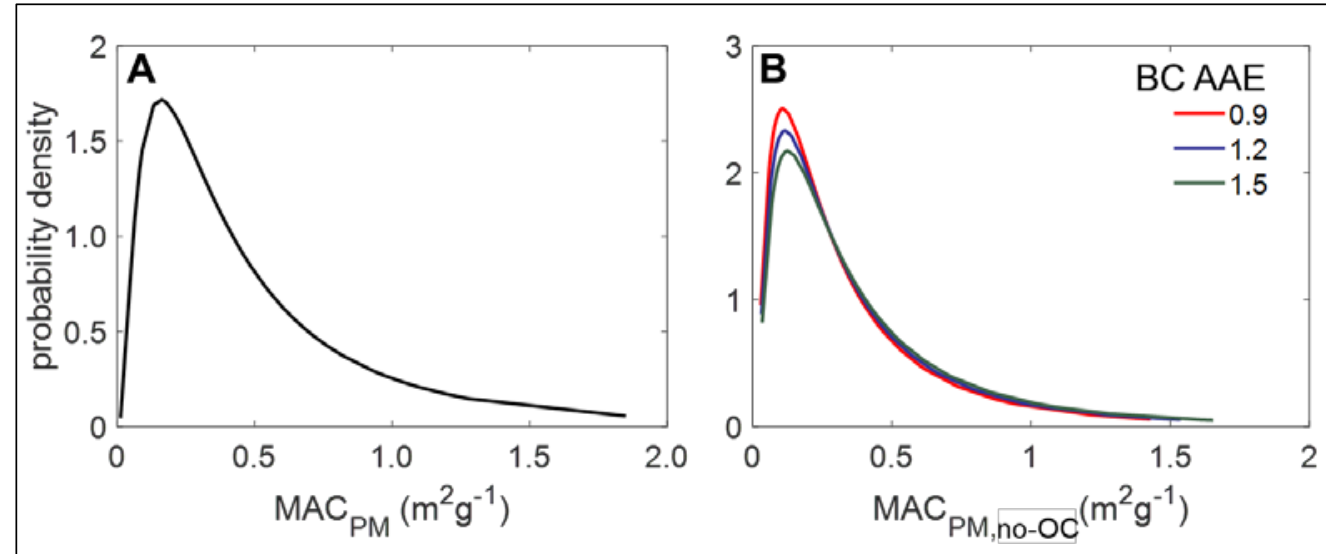

Fig. S4: Probability distributions of (A) $\mathrm{MAC}_{\mathrm{PM}}$ and (B) MAC $\mathrm{PM}_{\mathrm{PM} \text {, }-\mathrm{OC}}$ for all samples in this study.

Table S1: Slope (m), intercept (c) and adjusted $\mathrm{R}^{2}$ for the linear fits: $\mathrm{MAC}_{\mathrm{PM}, \mathrm{no}-\mathrm{OC}}=\mathrm{m}^{*} \mathrm{MAC_{ \textrm {PM } }}+\mathrm{c}$.

\begin{tabular}{|c|c|c|c|}
\hline & \multicolumn{3}{|c|}{ BC AAE } \\
\hline & 0.9 & 1.2 & 1.5 \\
\hline $\mathrm{m}$ & 0.82 & 0.88 & 0.94 \\
\hline C & -0.09 & -0.09 & -0.1 \\
\hline Adjusted $\mathrm{R}^{2}$ & 0.97 & 0.97 & 0.97 \\
\hline
\end{tabular}




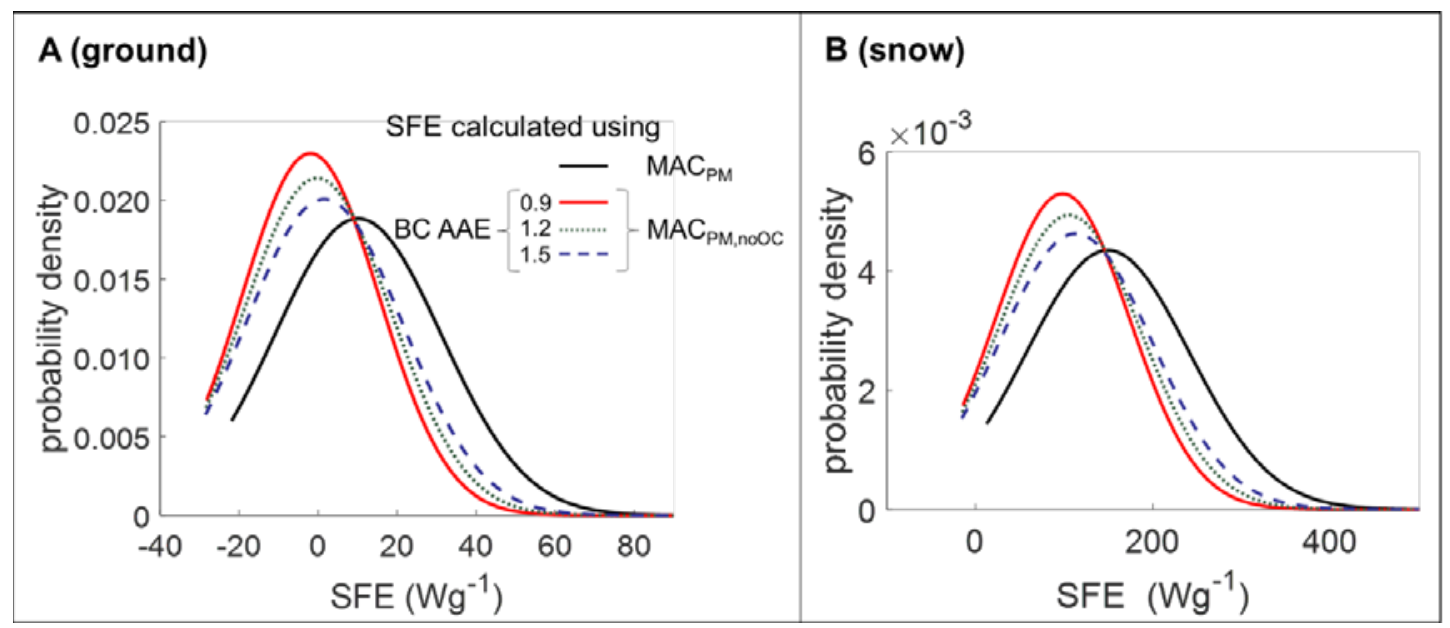

Fig. S5: Probability distributions of simple forcing efficiency of cookstove particulate emissions over (A) ground and (B) snow. Forcing was calculated with and without OC light absorption, with a fixed MSC $=1.5$ $m^{2} g^{-1}$.

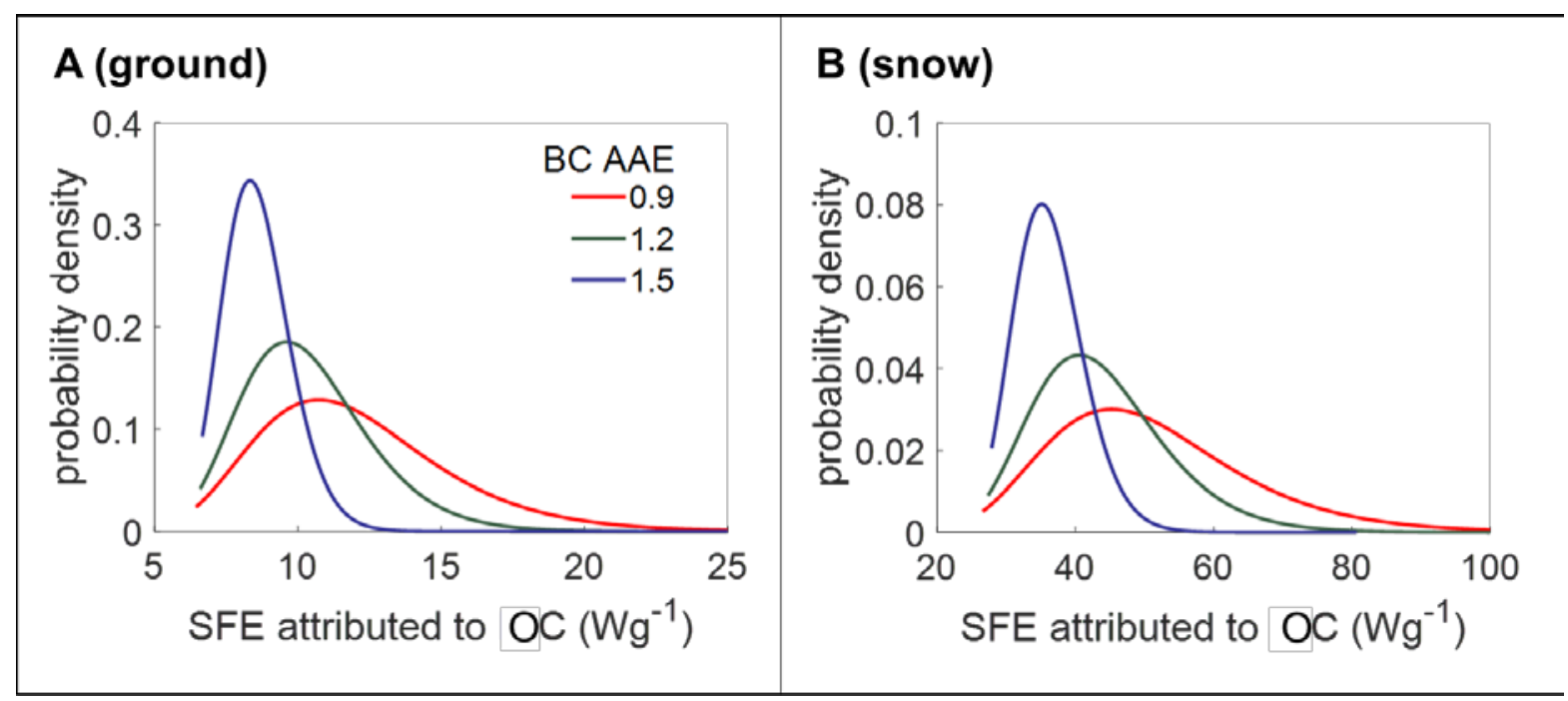

Fig. S6: Probability distributions of simple forcing efficiency of cookstove particulate emissions attributed to OC light absorption over (A) ground and (B) snow. The difference between SFE depends only the difference in the respective MAC values: $S F E_{P M}-S F E_{P M, n o o C}=S(\lambda) \tau_{a t m}^{2}(1-$ FC) $\alpha_{S} \times\left[M A C_{P M}(\lambda)-M A C_{P M, n o o C}(\lambda)\right]$ This difference can be interpreted as the contribution of OC light absorption to forcing by cookstove emissions. 


\section{References:}

1. Pandey, A.; Patel, S.; Pervez, S.; Tiwari, S.; Yadama, G.; Chow, J. C.; Watson, J. G.; Biswas, P.; Chakrabarty, R. K., Aerosol emissions factors from traditional biomass cookstoves in India: insights from field measurements. Atmos. Chem. Phys. 2017, 17, (22), 13721-13729.

2. Pandey, A.; Shetty, N. J.; Chakrabarty, R. K., Aerosol light absorption from optical measurements of PTFE membrane filter samples: sensitivity analysis of optical depth measures. Atmos. Meas. Tech. 2019, 12, (2), 1365-1373.

3. Chen, Y.; Roden, C. A.; Bond, T. C., Characterizing biofuel combustion with patterns of real-time emission data (PaRTED). Environ. Sci. Technol. 2012, 46, (11), 6110-6117. 